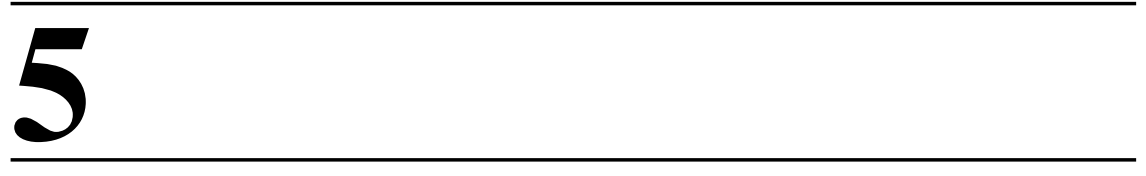

\title{
WOUND HEALING APPLICATIONS OF NANOMATERIALS
}

\author{
Berna Senturk, Gozde Uzunalli, Rashad Mammadov, \\ Mustafa O. Guler, and Ayse B. Tekinay \\ Institute of Materials Science and Nanotechnology, National Nanotechnology \\ Research Center (UNAM), Bilkent University, Ankara, Turkey
}

\subsection{INTRODUCTION}

The prevalence of diabetes and obesity, as well as the increase in the percent of the aging population, has resulted in a sharp rise in the costs of wound care, creating a major and so far unmet need for effective treatments against wounds requiring long-term medical attention. Chronic skin ulcers caused by diabetes mellitus, pressure, or venous stasis affect about 6.5 million individuals worldwide (Jones et al., 2002), while $15 \%$ of diabetic patients suffer from foot ulcers (DFUs) - a leading cause of limb amputations (Brem and Tomic-Canic, 2007). In addition to chronic and traumatic wounds, burn injuries also represent a leading cause of death: The World Health Organization (WHO) reported a total of 11 million severe burn cases in 2004, and 265,000 deaths per year are caused by burns (WHO, April, 2014).

Although nanomaterials are promising candidates for assisting the wound regeneration process, they nonetheless are a recent area of research, and considerable effort is required to develop nanomaterial-based treatments

Therapeutic Nanomaterials, First Edition. Edited by Mustafa O. Guler and Ayse B. Tekinay.

(C) 2016 John Wiley \& Sons, Inc. Published 2016 by John Wiley \& Sons, Inc. 
to the point that they surpass the current medical standard of care. The clinical challenges in using nanomaterials for wound healing include safety issues related to the materials, the requirement for new materials to induce vascularization more rapidly than their conventional counterparts, and issues related to the adequate promotion of cell attachment to the wound bed. It is hoped that the development of a new generation of biomaterials will overcome the clinical challenges and provide a more systematic and effective approach for wound management.

A better insight into wound injuries and the mechanism of their recovery may allow the design of more effective wound dressings, for example, by combining the hormones and growth factors required for cell migration and proliferation with scaffold materials that imitate the structure of the healthy extracellular matrix (ECM). However, a deeper understanding of the wound physiology is necessary to achieve this goal or to appreciate the current level of progress in artificial wound regeneration. As such, this chapter first provides an overview of skin structure and its biological response to the tissue loss in wound healing process and subsequently reviews the classical and next-generation scaffolds and materials that are used to minimize scar formation while accelerating the healing process. We also provide a snapshot of the natural and synthetic biomaterials currently in clinical use in this field.

\subsubsection{The Structure of Healthy Mammalian Skin}

By far the largest organ of the mammalian body, skin, encompasses the whole surface of the organism as a physical barrier against external factors such as infection, dehydration, and UV radiation. In addition to physical protection, it is also responsible for thermoregulation, sensory detection, and endocrine function (e.g., vitamin D synthesis, conversion of prohormones into hormones). The skin is assisted in its function by a variety of specialized structures, such as hair follicles, sweat glands, and sebaceous glands, collectively called skin appendages. As a result of its underlying epidermal stem cell population, it also displays an exceptional capacity for self-renewal under normal physiological conditions (Menon, 2002; Yildirimer et al., 2012).

The skin consists of two distinct layers: an outermost covering called epidermis and an inner region called dermis. The demarcation of skin layers is an issue of contention, and three layers (epidermis, dermis, and hypodermis) are sometimes proposed; however, the present chapter considers the skin to consist of the two aforementioned layers.

The epidermis is a thin (from 0.04 to $1.6 \mathrm{~mm}$ ) and avascular layer that consists mostly of specialized epithelial cells, such as keratinocytes, and 
nonepithelial cells, such as melanocytes, Langerhans cells, and Merkel cells. The keratinocytes of the stratified epidermis are wholly undifferentiated at the bottom of the epidermis, where they form a basal layer. These cells gradually move upward as they differentiate, eventually reaching the uppermost layers as squamous cells (stratum spinosum), granular cells (stratum granulosum), and cornified cells (stratum corneum). The epidermis is anchored to the dermis through hemidesmosomic connections to an interlayeric basement membrane. This membrane, which contains laminins, type IV collagen, perlecan, heparan sulfate proteoglycans, fibronectin, and nidogens, provides the interchange of nutrients between the two layers (Markeson et al., 2015; Menon, 2002). ECM components, such as collagen, elastin, proteoglycans, and glycosaminoglycans (GAG), and cellular structures, such as vessels, nerves, adipose cells, muscle fibers, glands, and hair follicles, form the inner layer of the skin, called the dermis. This region of connective tissue ranges from 1 to $4 \mathrm{~mm}$ in thickness and is composed of three major types of cells: fibroblasts, macrophages, and adipocytes (Markeson et al., 2015).

\subsubsection{The Mechanisms of Wound Healing}

Wound healing occurs in nearly all tissue types, and the order of events during the healing process is similar across different tissues. The regeneration of wounds is an interactive, complex, and dynamic process and starts immediately in response to tissue damage, which triggers the activation of a series of inter- and intracellular signaling pathways that ultimately culminate in the recovery of the anatomic continuity of the tissue. Following the initial step of blood clot formation, inflammatory pathways are activated for the secretion of specific soluble factors, and progenitor and stem cells undergo major metabolic changes to produce the cell populations required for the regeneration process (Gurtner et al., 2008). The tissue repair process is rapid, and the injured tissue is initially replaced with a poorly organized ECM that lacks skin appendages and is dissimilar to the tightly wound basket-weave structure of unwounded tissue. This "stopgap" approach results in the formation of a scar, and the injured tissue is not fully restored to its previous functionality following the regeneration process (Bayat et al., 2003).

Unlike the wound repair mechanisms of adult mammals, lower vertebrates (e.g., salamanders) are capable of total regeneration in organs such as limbs and eyes (Brockes et al., 2001). It is also known that mammalian embryos are able to undergo scarless healing (Ferguson et al., 1996). A suppressed or immature immune system appears to be a key necessity for regeneration to occur without scar tissue formation, though postnatal 
defects of liver and bone tissues have been reported to partially heal without scar formation (Gurtner et al., 2008).

The formation of scar tissue, although suboptimal in the long run, is evolutionarily advantageous: The ability to rapidly exclude opportunistic pathogens and partially restore tissue functionality outweighs the potential long-term disadvantages of scars. Nonetheless, the disadvantages inherent to the incomplete regeneration of wounded tissues are reflected in healthcare costs, especially in patients with metabolic disorders like diabetes or obesity, as well as those suffering from old age. Due to the limited human capacity for recovery, even otherwise healthy individuals may face lifelong disabilities if they receive severe injuries at a young age. However, a better understanding of the natural healing process and its limitations may allow the restoration of the original tissue structure and function in major wound or burn injuries, improving the life quality of patients and decreasing the economic burden by preventing the formation of scars. The process of wound repair has three distinct but spatially overlapping stages: inflammation, proliferation, and maturation.

5.1.2.1 Inflammation Inflammation is a crucial step that protects the organism against opportunistic pathogens and prepares the region of injury for the rest of the repair process. It is characterized by discomfort, loss of movement, edema, erythema, and heat. This phase proceeds for about $3-5$ days in acute wounds, while it is extended in chronic wounds (Gardner et al., 2001).

The healing process begins immediately following injury and is initiated by the activation of the coagulation cascade, which prevents the loss of blood from the injury site. A platelet plug is formed at the wound bed, creating a provisional scaffold for migrating cells and serving as a physical barrier to protect the wound from the external environment. The formation of the clot is achieved through the aggregation of fibrin with fibronectin, vitronectin, and thrombospondin-1 (TSP-1) (Martin, 1997). The activated platelets, which trigger the release of growth factors such as epidermal growth factor (EGF), platelet-derived growth factor (PDGF) (Lynch et al., 1987), and transforming growth factor $\beta$ (TGF- $\beta$ ) 1 and 2 (O'Kane and Ferguson, 1997), are also necessary in this step. Platelet cells also attach to the fibronectin matrix and release ECM proteins to further assist in the production of the blood clot.

Growth factors released by activated platelets, especially PDGF and TGF- $\beta$, are responsible for triggering the subsequent stage of inflammation. Neutrophils also arrive at the wound site in response to platelet degranulation, bacterial degradation products, and fibrin proteolysis. These cells are responsible for sterilizing the wound by killing opportunistic 
bacteria and secrete the cytokines that activate the resident populations of fibroblasts and keratinocytes (Gillitzer and Goebeler, 2001). Monocytes are also recruited to the wound site and are activated as macrophages. In the absence of excessive infection, neutrophils are phagocytosed by macrophages in response to the interaction between specific ECM proteins and integrin receptors. Another task of macrophages is the expression of important growth factors such as colony-stimulating factor-1 (which is responsible for the survival of monocytes and macrophages), PDGF, tumor necrosis factor- $\alpha$ (TNF- $\alpha$ ), insulin-like growth factor (IGF), interleukin-1 (IL-1), and some TGF family members. The lack of macrophage infiltration appears to result in impaired wound healing, while the treatment of scars with a TGF- $\beta 1$ inhibitor was shown to reduce inflammation and improve the dermal architecture of the scar tissue (Shah et al., 1995).

In addition to neutrophils and macrophages, mast cells and lymphocytes are also crucial for the wound healing process. The production of interleukin-4 by mast cells triggers the proliferation of fibroblasts, which in turn initiate the proliferation phase. Lymphocytes function both as immune effector cells and as growth factor sources; their secretions are associated with the remodeling process.

5.1.2.2 Proliferation The formation of new tissue is the second stage of wound healing, involving the deposition of a temporary ECM and the priming of the vascular network for the formation of new blood vessels. This step allows the isolation of the wound from external factors, further preparing the tissue environment and its constituent cells for the subsequent ECM remodeling process.

\subsection{Reepithelialization Wound injuries compromise the integrity} of the epidermal cover, and the injured dermis is therefore susceptible to damage from the external environment. Although a temporary barrier is provided by the blood clot, a more permanent protection is necessary for the later steps of wound repair to occur. Reepithelialization involves the formation of this protective layer and occurs through the migration, proliferation, and differentiation of local keratinocytes and epithelial stem cells from hair follicles or sweat glands (Lau et al., 2009; Martin, 1997; Miller et al., 1998). Two processes are required for this step: the dissolution of cell-cell and cell-basement membrane connections (Singer and Clark, 1999) and the activation of signaling pathways needed for the release of various growth factors and cytokines (Werner and Grose, 2003).

The integrity of uninjured epidermis is maintained by the hemidesmosomal links between keratinocytes and the basement membrane, as well as 
the desmosomal bridges that connect one keratinocyte to another. Wound injuries disrupt this structure and alter cell membrane permeability to ions by activating membrane-associated kinases, resulting in cell polarization through reorganization of intercellular filaments (Martin, 1997). However, keratinocytes in healthy tissue are still anchored to each other and the surrounding matrix, and their migration requires enzymatic cleavage of desmosomes and hemidesmosomes by collagenase and elastase. In addition to enzymatic cleavage, it is also known that the activation and relocalization of protein kinase $\mathrm{C} \alpha$ are important for the dissociation of desmosomal connections (Wallis et al., 2000).

Following migration, keratinocytes establish new epithelial layers at the wound edge by forming focal adhesions with the local integrin network. This process of dissociation and reanchoring is called as "keratinocyte shuffling" and allows keratinocytes to spread over the fibronectin-rich provisional matrix with the help of a gradient of cytokines and growth factors, such as IL-1, EGF, and TGF- $\alpha$ (Clark et al., 1982; Jiang et al., 1993). Keratinocyte shuffling is considered complete when each keratinocyte reforms its connections with its neighbors and reestablishes the intercellular hemidesmosome network (Jacinto et al., 2001).

The entire wound healing process is orchestrated by a complex cocktail of growth factors. EGF is one of the most important growth factors for the reepithelialization process, inducing the autophosphorylation of its receptor (EGF receptor (EGFR)) to promote cell proliferation (Martin, 1997) and migration (Rheinwald and Green, 1977). In addition, it has been demonstrated that a direct relationship exists between EGFR expression and epidermal thickness in wounded tissues (Stoscheck et al., 1992). Another important group is the fibroblast growth factor (FGF) family. It is known that FGF-2 is important for wound reepithelialization and keratinocyte motility (Bhora et al., 1995), while two other members of this family, FGF-7 (Marchese et al., 1995) and FGF-10 (Beer et al., 1997), are called keratinocyte growth factors and reported to be upregulated in skin wounds.

5.1.2.2.2 Angiogenesis Wound healing necessitates the coordinated activity of a large number of specialized cells, which must be supplied with oxygen and nutrients through a complex network of blood vessels. As such, angiogenesis in wound healing is a dynamic and multistep process, and several characteristics distinguish it from other forms of blood vessel generation. First, wound angiogenesis is unique in that it is activated by the immune response, although it is also regulated through the activity of endothelial cells, angiogenic growth factors, and ECM molecules. New blood vessels can be formed both by the sprouting of preexisting capillaries, which is 
called angiogenesis, and by the de novo production of bone marrow-derived endothelial precursors (EPCs), which is called vasculogenesis (Carmeliet, 2003; Eming et al., 2007a; Phelps and Garcia, 2009). Both occur during wound healing, and the processes are supported by one another.

The most important angiogenic factors are vascular endothelial growth factor (VEGF) and basic fibroblast growth factor (bFGF or FGF-2). Although a variety of cells can produce these factors, macrophages in particular are responsible for secreting FGF-2 and VEGF in response to the hypoxic environment of the wound bed (Greenhalgh, 1998). It is also known that damaged endothelial cells secrete FGF-2, while VEGF is released by keratinocytes (Martin, 1997). New vascular formation requires the degradation of the old ECM in order to create space for new tubules, and endothelial cells also perform this function by secreting a plasminogen activator to combine plasmin and procollagenase into active collagenase. The degradation of the basement membrane allows production of new vessels at the wound bed, and angiogenic growth factor secretion ceases when enough oxygen is supplied to the wound area (Bishop, 2008).

\subsection{Granulation Tissue Formation The formation of granulation} tissue, or new stroma, is a transition step between the proliferation and remodeling phases. This tissue invades into the fibrin/fibronectin-based provisional matrix about 4 days after injury. Its granular appearance is a result of the large numbers of capillaries, macrophages, fibroblasts, and collagen bundles present within its matrix (Singer and Clark, 1999).

This tissue is formed as a result of macrophage activity. In particular, macrophage-secreted growth factors, such as platelet-derived growth factor 4 (PDGF-4) (Heldin and Westermark, 1999) and TGF- $\beta 1$ (Beck et al., 1991), stimulate the resident fibroblast population into secreting a new ECM that contains collagen type III, fibronectin, GAG, proteoglycans, and hyaluronic acid (Eckes et al., 2010). Over time, some fibroblasts differentiate into $\alpha$-smooth muscle actin ( $\alpha$-SMA)-expressing myofibroblast cells (Darby et al., 1990). These cells form the contractile apparatus of the wound site and are characterized by large actin bundles, as well as by $\alpha$-SMA. The contractile ability of these cells introduces a mechanotransduction element into the wound ECM. In addition, myofibroblasts can use the transmembrane connections between their intracellular actin and extracellular fibronectin domains (a system collectively called the fibronexus) to transmit their contractions across the tissue ECM (Gabbiani, 2003). Through their collective contractions, myofibroblasts may therefore physically move the wounded tissue area. This, in conjunction with the reepithelialization process, allows the closure of the wound. 
5.1.2.3 Tissue Remodeling Remodeling or resolution is the last phase of the healing process, starting at day 21 and lasting for a year or more after injury. It is the phase of regression in which the capillary- and cellrich granulation zone is replaced with a more mature wound tissue. Myofibroblasts, endothelial cells, and macrophages undergo apoptosis during this stage, and the new tissue only contains a collagen-rich ECM and a sparse population of cells (Greenhalgh, 1998). In addition, although a large number of capillaries are required to supply the metabolic demands of rapidly proliferating, migrating, and differentiating cells during the tissue repair process, these blood vessels are no longer required by this point. As such, the wound vascular density decreases to normal during this phase.

Another major activity at this stage is the production of a new collagen matrix. Collagen type III, which consists of small parallel bundles, is the dominant component of the ECM during the early stages of wound repair. As the wound matures, collagen III is replaced by a stronger type of collagen, collagen I, which forms thin fibrils and is the collagen type normally found in healthy skin (Lovvorn et al., 1999). This replacement is called as collagen deposition and under the control of matrix metalloproteinases (MMPs), which are secreted by endothelial cells, fibroblasts, and macrophages (Levenson et al., 1965). In tandem with the controlled degradation and redeposition of ECM proteins, the maintenance of tissue homeostasis in the skin is provided through cytokine-regulated mesenchymal-epidermal interactions (Szabowski et al., 2000).

Although the wound is considered healed at the end of the remodeling phase, damaged tissue nonetheless does not immediately regain its former strength. About $30 \%$ of the skin's breaking strength is lost following wounding and repair, which is attributed to the presence of a stronger collagen network in healthy tissue, laden with intermolecular crosslinks (Levenson et al., 1965).

\subsubsection{Repair Process in Chronic Wounds}

Factors such as type I diabetes, neuropathies, stress, and aging can interfere the natural healing process, resulting in the formation of long-term injuries called chronic wounds. These injuries are generally classified under three groups: diabetic ulcers, arterial and venous ulcers, and pressure ulcers. In all three types, the wound healing process is initiated through the formation of a blood clot, as is typical for the repair of normal skin injuries. However, the inflammation period is prolonged, and the subsequent repair of the damaged tissue area is delayed in chronic wounds (Eming et al., 2007b). 
In addition, the production/degradation balance seen in natural wound healing is heavily disrupted in favor of degradation in chronic wounds, and the wound area is saturated with an elevated concentration of proteases that hinder the activity of ECM components, growth factors, and/or their receptors (Mast and Schultz, 1996).

Venous ulcers generally occur due to the incompetence of venous valves, which results in the pooling of blood within the vein and increases the pressure it experiences. This increased pressure allows blood proteins, especially fibrin, to leak into the extravascular space, preventing regular blood flow within the vein and creating a sink for growth factors required for wound healing (Walker, 1999). These effects, in turn, decrease collagen production within the wound area (Pardes et al., 1995). Arterial ulcers, in contrast, typically occur as result of narrowed or ischemic arteries. As the arterial blood flow is blocked, the wound area cannot be supplied with an adequate amount of oxygen, nutrients, and growth factors, which delays the wound healing process (Bonham, 2003).

Diabetes is another major cause of chronic wounds, as the neural and vascular pathologies experienced by diabetic patients may often impair the wound healing process. Hyperglycemia triggers the formation of advanced glycation end products, which have been reported to facilitate the formation of abnormal granulation tissue (Goldin et al., 2006). In addition, it is known that the metabolism of nitric oxide, a major factor in angiogenesis, is altered in diabetic patients (Linden et al., 2008). MMPs, a group of proteins involved in ECM degradation, also show increased activity in diabetic patients: Although the remodeling of the wound area is necessary for the repair process, these enzymes must be regulated through tissue inhibitors of metalloproteinases (TIMPs), and the balance between these two factors is compromised in diabetic conditions (Lobmann et al., 2002).

Pressure ulcers, or bedsores, occur when shearing force and/or pressure is applied to the soft tissues over long periods of time, especially in patients with limited mobility. Over time, shear force and pressure trigger reperfusion injury, ischemia, and, eventually, tissue necrosis (Defloor, 1999).

\subsection{APPLICATIONS OF NANOMATERIALS FOR THE ENHANCEMENT OF WOUND HEALING PROCESS}

The development of biodegradable and nontoxic materials for the enhancement of the wound healing process is an active area of research, and advances in nanotechnology have contributed greatly to the design 
efficiency of such materials. Nanobiomaterials allow the design of wound dressings that not only create a suitable biomolecular environment for the regeneration process to occur but also protect against infections at the wound site and facilitate the controlled release of bioactive materials such as growth factors.

Natural and synthetic polymers, such as polysaccharides (e.g., alginate, chitin, chitosan, heparin, chondroitin), proteoglycans, and proteins (e.g., collagen, gelatin, fibrin, keratin, silk fibroin, eggshell membrane) are commonly used in wound management, as those nanomaterials display low toxicities and can be naturally degraded over time in the body. The large surface-to-volume ratios inherent to nanoscale materials is another major advantage and allows a small volume of material to contain a large amount of therapeutic cargo. Advances in the design of soft materials, such as hydrogels and bioscaffolds, have revolutionized the field of wound management research, as these materials can be engineered to provide both the biophysical environment and biochemical signals necessary for the wound regeneration process.

Wound dressings protect the damaged tissue against opportunistic pathogens and assist the recovery of dermal and epidermal tissues. The choice of dressing material depends on the cause and type of the wound, with both natural and artificial dressings being available.

\subsubsection{Artificial Skin}

An "artificial skin" that can adequately replace damaged skin tissue is the ideal type of wound dressing, and considerable effort has been spent on developing such a material. Natural skin transplantation is the first choice for clinicians and surgeons to replace damaged skin in severe burn injuries; though only large and deep wounds warrant this procedure (skin grafting is generally not necessary for first- and second-degree burns, which heal with little to no scarring). On the other hand, the wound bed in the thirddegree burns must be covered as quickly as possible with artificial or natural grafts (Centanni et al., 2011). Donor areas for natural skin transplantation include the chest, thighs, buttock, abdomen, or behind the ear. As the donor region must contain healthy skin, this procedure is not recommended for elderly patients with pressure sores and people with diabetic or other chronic ulcers. The treatment of these patients therefore necessitates the development of tissue engineering techniques for the artificial replacement of the skin.

Artificial tissues generally incorporate three main elements: a cell type, a differentiation-inducing substance, and a material matrix. Skin substitutes 
might be biological (e.g., xenografts, allografts, autografts, and amniotic substitutes) or synthetic (Halim et al., 2010). Xenografts are skin substitutes harvested from animals and serve as a temporary insulating layer during early stages of wound healing in humans. Allotransplantation, which refers to the transplant of substitute skin from the same species, allows a potential means of rapid intervention in burn wound management. Cadaveric skin allografts are one of the most common biological substitutes worldwide. There are two main strategies to preserve cadaveric skin allografts: cryopreservation and glycerol preservation. Glycerol has antibacterial and antiviral effects and is more cost efficient for the long-term storage and long-distance transport compared to cryopreserved skin. Amniotic substitutes derived from placenta of selected and screened donors are rich in collagen and various growth factors that support the healing process, thus improving wound closure rates and reducing scar formation (Halim et al., 2010).

Although these naturally derived skin substitutes are widely used in clinics, they cannot facilitate the complete regeneration of the skin due to limited donor sites, risk of infection, slow healing rates, and, in the case of autografts, the requirement to create new wounds to acquire the required skin tissue. As such, there is a substantial need for tissue-engineered skin constructs that support the complete regeneration of wound injuries. The development of artificial skin tissue may also enhance the scope of regenerative medicine, and techniques used in artificial skin production may be expanded for the generation of more complex structures, such as artificial organs. Additionally, advances in the design of therapeutic agents will no doubt also lead to a greater understanding of the pathophysiology of the wound healing process.

\subsubsection{Natural Nanomaterials for Wound Healing}

Natural materials are required to exhibit certain characteristics to be considered suitable for use as wound dressings. Foremost important among these criteria is the ability to serve as an adequate matrix scaffold for the cell types involved in the wound healing process (including stem cells, macrophages, fibroblasts, and/or epithelial cells). Also important is the ability to incorporate vital molecular signaling elements such as growth factors and signaling molecules. These functions allow the nanomaterial dressing to reduce inflammation, scar formation, and infection; and they may also play a role in various other processes. A large number of natural materials, such as collagen, gelatin, laminin, and chitin/chitosan, have been used as electrospun scaffolds for tissue engineering. 
5.2.2.1 Collagen Collagen is the major protein of the ECM and acts as a structural scaffold in various tissues (Lodish et al., 2000). Types I and III are the main types of collagen found in skin tissue. Collagen subunits pack together to form long, thin fibrils, which typically assume a triple helical structure. Triple helices of type I collagen consist of two $\alpha 1$ (I) chains and one $\alpha 2(\mathrm{I})$ chain, while type III collagen is composed of three $\alpha 1$ chains. Collagen fibers in the skin are mainly synthesized by fibroblasts and myofibroblasts. Collagen deposition occurs at the wound site following the third day postinjury and involves the replacement of type III collagen with type I. The replacement process gradually increases the stiffness and tensile strength of the regenerating tissue, as the tensile strength of type I collagen is greater than that of type III collagen.

It is known that collagen not only serves a structural support but also contributes to matrix deposition and cellular differentiation and migration during the wound healing process. A number of collagen dressings have been reported in the literature, often incorporating additional materials such as alginate (to control material efflux from the scaffold) and silver nanoparticles (AgNPs) (to provide antimicrobial effects). Some of these designs are commercially available and frequently used in surgical or clinical procedures (Fitzgerald and Steinberg, 2009).

Apligraf (Graftskin) (Organogenesis/Novartis, Canton, MA) is a wound dressing product that contains allogeneic neonatal foreskin fibroblasts and keratinocytes in bovine collagen gel; it is used in the treatment of chronic foot and venous leg ulcers. Cellular-allogeneic OrCel (Ortec International, New York, NY) contains both fibroblasts and keratinocytes from neonatal foreskin, cultured in a type I collagen sponge. It is used for treatment of split-thickness donor sites in patients with burn and surgical wounds in epidermolysis bullosa (Supp and Boyce, 2005). Cultured skin substitutes, composed of collagen-GAG substrates containing autologous fibroblasts and keratinocytes, facilitate permanent wound closure in burn injuries and congenital nevus and chronic wounds (Boyce et al., 1993).

The porous structure, high permeability, hydrophilicity, stability, and cell-supporting nature of collagen make this material a popular choice for wound dressings. In addition, the development of new material combinations of collagens and engineered skin substitutes may further enhance the utility and versatility of wound dressings in clinical use.

5.2.2.2 Laminin Laminin is a widely expressed protein that contributes to the formation of the ECM, particularly the basement membrane. Laminins comprise a family of at least 15 large trimeric basement membrane proteins, each of which is composed of one $\alpha$ chain, one $\beta$ chain, and 
one $\gamma$ chain and may display a distinct, tissue-specific biological role (Bosman and Stamenkovic, 2003). Laminin is critical for the cell-material interactions that occur during the wound healing process, and displays the ability to promote cell proliferation and attachment. Defects on laminin expression have been correlated with delayed or impaired wound closure. Junctional epidermolysis bullosa (JEB) is an inherited disease affecting laminin and collagen expression and characterized by fragility of the skin and mucous membranes. It has been shown that laminin 5 is highly expressed in migrating keratinocytes in the wound bed (Larjava et al., 2000). Due to its promotion of cell proliferation and attachment, lamininderived peptides are also used as wound dressing (Hashimoto et al., 2004; Malinda et al., 2008). In the peptide-based approach, topical application of laminin-derived peptides was shown to increase reepithelialization and granulation tissue formation in the early stages of wound healing in rats (Malinda et al., 2008). Similarly application of peptide conjugated scaffolds to the wound area, for example, the PPFLMLLKGSTR peptide derived from LG3 domain of $\alpha 3$ laminin, increased rates of skin reepithelialization (Min et al., 2010).

5.2.2.3 Chitin and Chitosan Chitin and chitosan are natural polysaccharides that display suitable biological and physicochemical characteristics for use as wound dressings. Their main advantages are oxygen permeability, biodegradability, and biocompatibility of these materials, as well as easily processability into hydrogels, fibers, membranes, scaffolds, and sponges. Chitin is an inexpensive material obtained from the exoskeleton of invertebrates. Chitosan is composed of randomly distributed $\beta$-(1-4)-linked D-glucosamine and $N$-acetyl-D-glucosamine units. After depolymerization, $N$-acetyl-D-glucosamine units induce fibroblast proliferation, critical for collagen deposition, and stimulate natural hyaluronic acid synthesis, which leads to faster wound healing and scar prevention.

Chitin-nanosilver composite scaffolds were found to possess excellent antibacterial activity against bacterial infection during the wound healing process (Jayakumar et al., 2010). Chitin and chitosan can also be used as slow-release drug delivery vehicles for growth factors to further accelerate the wound healing process (Dai et al., 2011).

5.2.2.4 Alginate-Based Nanomaterials Alginates are unbranched polysaccharides extracted from brown algae. Alginate-based wound dressings display hemostatic properties and are commonly used in bleeding wounds and burns. Materials such as calcium alginate, calcium sodium alginate, 
collagen/alginate, and gelatin/alginate are highly absorbent natural fiber dressings and can be obtained at minimal cost from processed algae. The high absorption capability of alginate facilitates the creation of a moist environment that promotes the formation of granulation tissue during the wound healing process (Mogoşanu and Grumezescu, 2014). The high water absorption capacity of alginate also helps to reduce blood loss. Chemical modifications with calcium and zinc can also be performed to improve the efficiency of alginate over conventional cotton wound dressings.

\subsubsection{Synthetic Nanomaterials for Wound Healing}

Synthetic materials allow greater control over properties such as porosity, controlled release capacity, biodegradability, and structural versatility, in addition to avoiding issues inherent to natural materials such as batch-tobatch variance and viral, bacterial, or other types of biological contamination. As such, they have been commonly used in the design of novel regenerative scaffolds that aim to provide optimum conditions for regeneration with high reproducibility. Increased control over material structure and composition is a major advantage for synthetic skin substitutes, the ultimate goal of which is to produce an ideal skin substitute that provides an effective and scar-free wound healing.

5.2.3.1 Carbon Nanotubes Carbon nanotubes (CNTs) are currently under consideration for clinical applications regarding wound healing, although there are serious concerns over the toxicity of these materials. CNT-based antiseptic bandages were produced by combining povidone-iodine with single-walled carbon nanotubes (SWNTs) (Simmons and Linhardt, 2012), and it has been shown that the presence of the multiwalled carbon nanotubes (MWCNTs) positively affects the wound healing process by decreasing the expression of genes that interfere with the function of the cytoskeleton, such as actin stress fibers (Zhang et al., 2011).

5.2.3.2 Hydrogels Hydrogels promote granulation, epithelialization, and autolytic debridement at the wound site by providing a moist environment that is conductive to the regeneration process. Commercially available hydrogel dressings are generally found in the form of membranes and sheets. Studies involving in situ formation of hydrogel wound dressings based on gelatin, oxidized alginate, and borax suggest that the application of hydrogels enhance the wound healing process in rat models (Balakrishnan et al., 2005). 
Full-thickness wounds dressed with a combination of chemically crosslinked GAG hydrogels and the commercial wound dressing Tegaderm ${ }^{\mathrm{TM}}$ displayed a significant increase in reepithelialization at days 5 and 7 compared to wounds treated with Tegaderm alone, suggesting that modified hydrogels can improve the efficiency of treatments based on conventional wound healing agents (Kirker et al., 2002). Hydrogels can also be improved through incorporation of drugs or growth factors.

5.2.3.3 Antimicrobial AgNPs The antimicrobial properties of silver have been known and utilized for centuries, and the material has lately been used in wound dressings for the same purpose. AgNPs with diameters below $100 \mathrm{~nm}$ display increased antimicrobial activity due to their larger combined surface areas. Several methods exist for the incorporation of AgNPs into wound dressings. The antibacterial properties of silver are well known; however, the safety of using AgNPs and their fate within the body or the environment should be addressed.

\subsubsection{Wound Dressings Containing Growth Factors}

Growth factors are soluble secreted proteins capable of affecting a variety of cellular processes involved in tissue regeneration. However, their clinical applications are limited due to the absence of robust delivery systems and biomaterial carriers in addition to the risks associated with carcinogenicity of these materials. Nonetheless, growth factors have been found to speed the healing of acute and chronic wounds in humans, and some are approved for clinical use by the FDA. The PDGF analog REGRANEX ${ }^{\mathrm{TM}}$, for example, is currently used for the treatment of leg and foot ulcers in diabetic patients (Chan et al., 2006). Endothelial growth factor (EGF) is another major factor in wound regeneration and has been reported to play a role in keratinocyte migration, fibroblast proliferation and differentiation, and granulation tissue formation (Yildirimer et al., 2012). In addition to its rapid breakdown within the wound environment, EGF has a mitogenic effect upon cells (Yildirimer et al., 2012).

Among VEGF family composed of VEGF-A, VEGF-B, VEGF-C, VEGF-D, and placenta growth factor, in particular VEGF-A is highly expressed in acute wounds and promotes angiogenesis. The main initiator of expression of VEGF is hypoxic environment. It has been showed that VEGF-A improves reepithelialization of diabetic wounds via enhancement of vessel formation (Galiano et al., 2004).

bFGF is another main growth factor responsible for the induction of angiogenesis, which is the most critical step during wound repair. It has 
been shown that the treatment of rabbit ears with exogenous bFGF accelerates wound healing compared to nontreated control; however, no increase was observed in capillary density (Komori et al., 2005).

Although topical application of growth factors is used for the treatment of chronic wounds, these materials are released in a noncontrolled manner if not encapsulated within a controlled delivery matrix. Slow release is required for the effective delivery of these polypeptides to the wound site, and matrix-encapsulated growth factors will better enhance the wound regeneration process.

\subsubsection{Biomimetic Materials}

Recent advances in material sciences and polymer chemistry have resulted in the development of biomimetic peptide fibers, an entire new class of nanomaterials that can be used to assist in wound regeneration. The ECM is an enormously complex network of peptides, proteoglycans, growth factors, and soluble proteins, and its components play critical roles at each and every step of the wound healing process. The dysfunction of any of these components may result in delayed wound healing, as is observed in the chronic wounds of hyperglycemic patients. Development of biomimetic scaffolds that restore or replace the roles of ECM components may therefore allow the treatment of slow-healing dermal injuries that are currently difficult to treat using conventional approaches.

A bioengineered fibronectin matrix-mimetic substrate was shown to induce cell migration and stimulate high rates of cell proliferation (Roy et al., 2011). Similarly, collagen-mimetic peptides that assume a nanofibrous triple-helix formation were effectively used to enhance the thrombosis process (Kumar et al., 2014). Mesenchymal stem cell (MSC)-seeded biomimetic collagen hydrogels significantly enhanced angiogenesis compared to MSC injection alone (Rustad et al., 2012).

The main fabrication techniques to engineer such materials mimicking the nanofibrous ECM proteins are electrospinning, self-assembly, and phase separation. Development of those techniques allows the design and fabrication of biomimetic scaffolds that offer tremendous potential applications in wound healing of the skin. The development of novel biomimetic scaffolds capable of promoting and directing new vascular network growth would eliminate a critical problem in tissue engineering, serving as an efficient solution to the problems associated with chronic wound healing. Future research is expected to yield more information on the unique properties of biomimetic scaffolds. 


\subsubsection{Current Challenges in the Design of Nanomaterials for Chronic Wound Management}

The WHO reports that 347 million people suffer from diabetes worldwide and expects the disease to be the seventh leading cause of death in 2030 . The incidence of diabetes has been on the rise and may soon become one of the foremost public health challenges that the world will face in the decades ahead. Factors such as age, obesity, malnutrition, and vascular disease may contribute to wound infection and delayed wound healing in diabetic patients. The metabolic effects of diabetes, combined with reduced blood flow and neuropathies (nerve damage) associated with the disease, increases the chance of foot ulcers and the severity of wound infections, potentially leading to limb amputation and even death. The design of smart scaffolds as wound dressing is therefore necessary to increase the quality of life in patients suffering from diabetic ulcers and similar chronic wounds.

Due to the variations between the pathophysiologies of wound and burn injuries, it is difficult to develop an artificial dressing material that can facilitate optimal healing of all types of skin damage. The initial assessment of chronic wounds is therefore essential before deciding on wound dressing choice, which should be made by taking a full account of the wound and dressing characteristics. The choice of dressing should be based on wound size, depth, severity of infection, and location. Patients with severe infection should use an appropriate antibiotic therapy; additionally, the wound can be treated with silver and iodine products (Moura et al., 2013, Table 5.1).

\subsubsection{Nanomaterials Used in the Treatment of Diabetic Wounds} Increase in the number of patients with delayed diabetic wound healing has made developing appropriate wound dressings for diabetic wounds an urgent necessity. Decreased reepithelialization and growth factor expression, a dysfunctional ECM, and impaired angiogenesis are the main major problems that interfere with the recovery of diabetic wounds.

Studies involving the local application of growth factors such as human epidermal growth factor (hEGF) have shown that treatment with high concentrations of hEGF is effective in promoting wound healing in diabetic foot injuries (Tsang et al., 2003). It has been shown that recombinant hEGF immobilized in a copolymer of poly( $\varepsilon$-caprolactone) (PCL) and poly(ethylene glycol) (PEG) may increase the proliferation and phenotypic expression of keratinocytes (Choi et al., 2008). Although materials such as collagen and hydrogels have been used to facilitate the healing of diabetic wounds, dressings that can encapsulate growth factors or other materials that induce angiogenesis are more favorable for this purpose. 
TABLE 5.1 Commercially Available Wound Dressings and Their Recommended Regions of Application

\begin{tabular}{|c|c|c|c|}
\hline Product Name & Type & Usage & Reference \\
\hline $\mathrm{AQUACEL}^{\circledR}$ & Hydrofiber & Chronic wounds & $\begin{array}{l}\text { Cohn et al. } \\
\text { (2004) }\end{array}$ \\
\hline AQUACEL Ag & $\begin{array}{l}\text { Hydrofiber } \\
\text { containing } \\
\text { silver }\end{array}$ & $\begin{array}{l}\text { Leg ulcers, } \\
\text { superficial } \\
\text { pressure ulcers, } \\
\text { partial-thickness } \\
\text { burns }\end{array}$ & $\begin{array}{l}\text { Barnea et al. } \\
\qquad(2010)\end{array}$ \\
\hline $\begin{array}{l}\text { REGRANEX }^{\circledR} \\
\text { (FDA approved) }\end{array}$ & $\begin{array}{l}\text { Gel containing } \\
\text { platelet-derived } \\
\text { growth factor } \\
\text { (PDGF) }\end{array}$ & $\begin{array}{l}\text { Chronic and } \\
\text { traumatic wound }\end{array}$ & $\begin{array}{c}\text { Chan et al. } \\
\text { (2006) }\end{array}$ \\
\hline OASIS $^{\circledR}$ & $\begin{array}{l}\text { Derived from } \\
\text { porcine small } \\
\text { intestinal } \\
\text { submucosa } \\
\text { (SIS) }\end{array}$ & $\begin{array}{l}\text { Partial- and } \\
\text { full-thickness } \\
\text { wounds, pressure } \\
\text { ulcers, venous } \\
\text { ulcers, chronic } \\
\text { vascular ulcers, } \\
\text { diabetic ulcers, } \\
\text { trauma wound }\end{array}$ & $\begin{array}{c}\text { Mostow et al. } \\
\text { (2005) }\end{array}$ \\
\hline $\begin{array}{l}\text { SANTYL }^{\circledR}(\text { FDA } \\
\text { approved })\end{array}$ & $\begin{array}{l}\text { Ointment, } \\
\text { biological } \\
\text { enzymatic } \\
\text { debriding agent }\end{array}$ & $\begin{array}{l}\text { Chronic dermal } \\
\text { ulcers, severe } \\
\text { burns }\end{array}$ & $\begin{array}{l}\text { Hansbrough } \\
\text { et al. } \\
\text { (1995) }\end{array}$ \\
\hline IODOFLEX $^{\circledR}$ & $\begin{array}{l}\text { Cadexomer } \\
\text { containing } \\
\text { silver }\end{array}$ & Chronic wounds & $\begin{array}{l}\text { Bianchi } \\
\quad(2001)\end{array}$ \\
\hline
\end{tabular}

The encapsulation of growth factors in a polymer matrix not only protects the growth factor against enzymatic degradation within the body but also allows its release into a localized area at a controllable rate. As such, current strategies for the treatment of diabetic wounds mainly involve administration of materials that provide controlled release of growth factors. Since decreased angiogenesis contributes to impaired tissue repair, VEGF is also commonly incorporated into scaffolds intended for use in the treatment of diabetic wounds (Galiano et al., 2004). In addition to delivery of growth factors, engineered biomimetic materials such as heparan sulfate have shown an enhanced wound healing prowess compared to unloaded scaffolds (Garcia-Filipe et al., 2007). 


\subsubsection{Nanomaterials Used for the Treatment of Peripheral} Arterial Disease Peripheral arterial disease (PAD) is an atherosclerotic disease that is commonly seen in the elderly population. Diabetic PAD patients experience a gradual reduction in the delivery of oxygen- and nutrient-rich blood to the legs, which results in severe infections, osteomyelitis, and a higher risk of lower leg amputations (Lipsky et al., 2012; Reiber et al., 1999). PAD patients who also present with foot ulcerations tend to display other characteristics, such as neuropathic ulcers (Prompers et al., 2008). These ulcers are commonly located at the pressure points on the foot, mostly at the tips of the toes.

The lack of suitable natural materials and limitations of synthetic materials for treatment of PAD patients necessitate discovery of new routes for effective clinical therapy. Combinations of proper dressings with endothelial progenitor cells (EPC) are one important and exciting advance in the field of tissue engineering.

\subsection{PEPTIDE NANOFIBER GELS FOR WOUND HEALING}

Numerous materials for wound healing were mentioned in previous sections. Salient features of these materials are designed in an attempt to provide a desired niche for robust wound healing process. Fibrous structures of these materials serve as a scaffold for incoming cells. Growth factors or other bioactive molecules can be encapsulated or conjugated into materials to provide immigration of cells with necessary biological signals. Cell adhesion epitopes can be included in the material to facilitate cell attachment at damaged site. Mechanical properties of the materials should be cognate to the tissue they will function in. Besides these materials should be biodegradable and biocompatible. However, to gather all these properties in desired quality in one material is a formidable task. Obstacles here include synthesis of the fibrous materials with fibers of nanoscale size, conjugation of the bioactive elements onto them, and controlling their stiffness. On the other hand, nanofibrous materials formed by self-assembly of peptide amphiphile (PA) molecules have necessary features in remarkable quality. Nanofibers with a few nanometers in diameter and micron length can be obtained easily by this way. Their peptide part allows versatility in peptide epitopes and also provides easy way of addition of other chemical functional groups. Their structures are also comprised of biodegradable and biocompatible elements. 


\subsubsection{Relevance of Nanofibrous Structure of Peptide Gels for Wound Healing}

5.3.1.1 Bioactive Epitopes ECM proteins such as laminin and fibronectin induce signaling pathways in cells through their specific epitopes. Induction of these signaling pathways is very critical for various cellular processes such as proliferation, differentiation, as well as organization of the cells in tissues. ECM proteins are evolved to carry various domains charged with different functions, which allows them to operate in different contexts. Our body is adapted to perform in complex environments with scarce resources and has high levels of sophistication, which allows it to respond appropriately to an enormous variety of environmental signals. Since resources to build its structure are scarce, economy principle imposed itself on the design of our body. Accordingly resources to build our intricate body are distributed so that it can respond to millions of adverse conditions. However, when it comes to triggering specific signaling pathway involved in certain repair mechanism, we only need the appropriate epitopes of specific proteins. Scale of response provided by our body in natural environment may not be optimal in each case since distribution of resources occurs according to the distribution of signals in natural environment where mammalian body has evolved. Materials that present these epitopes with artificially high density unlike to the natural ECM proteins would be more valuable for providing the optimal response to certain disease conditions. For example, the peptide nanofibers carrying IKVAV epitopes showed better capability of inducing neuronal differentiation than laminin proteins from which IKVAV epitopes derived (Silva et al., 2004). Here, PA design allowed to achieve presentation of IKVAV epitopes on them with enormously high density. PA nanofibers are also amenable to change concentration of epitopes for optimal arrangement of signals on nanofibers for cells (Shah et al., 2010).

5.3.1.2 Topographical Cues Topography of the extracellular environment influences cell shape and behavior extensively. Size and type of patterns on substrates affect cell proliferation, migration, and differentiation (Kim et al., 2012). Basement membrane of epithelial tissue has a complex 3D texture in the nanometer range (Kim et al., 2012). Nanofibrous architecture also has been shown to facilitate cell attachment through enhanced protein absorption (Woo et al., 2003). PA form 3D nanofiber network (Fig. 5.1), which resembles the architecture of natural ECM that contact with epithelial and dermal cells. Pore size of network also can be controlled via changing concentration of PA molecules, which provide suitable topographical cues for wound healing applications. 


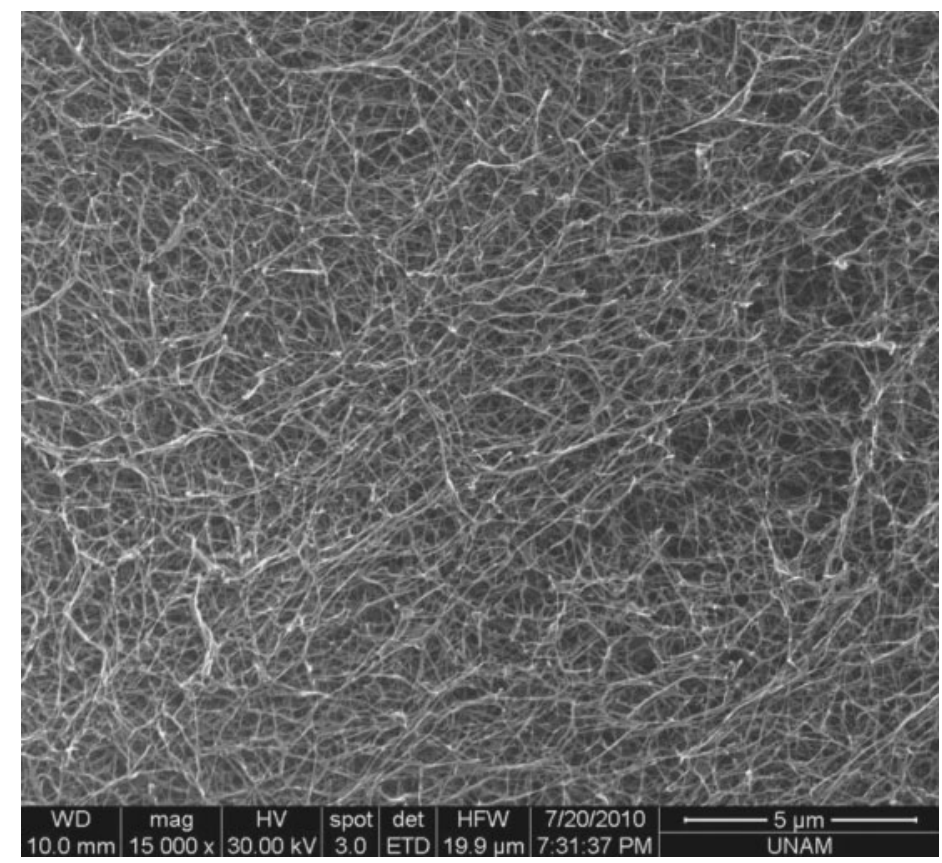

FIGURE 5.1 Three-dimensional nanofibrous network formed by peptide amphiphile nanofibers.

\subsubsection{Engineered PA Nanofiber Gels for Wound Healing and Insights into Various Designs}

5.3.2.1 Growth Factor Binding An enormously complex growth factor network takes role in wound healing mechanism (Werner and Grose, 2003). These growth factors regulate physiological processes such as angiogenesis (e.g., VEGF and FGF-2) and reepithelialization (e.g., EGF, HGF, and TGF- $\beta$ ), which are critical for repair process (Gurtner et al., 2008). Conventional approaches in tissue engineering focus on encapsulation of growth factors in scaffold systems to provide signals for cells. Burst release of growth factors from these materials became a big challenge with these designs since it causes aberrant regeneration besides inefficient expenditure of expensive growth factors. In order to overcome this, growth factors were conjugated to materials either noncovalently or covalently. Interaction of growth factors with the scaffold materials slows the release of growth factors that protect them from degradation and provides a signaling niche for cells inside the material. In order to conjugate growth factors to materials, the latter can be decorated with various binding sites. Indeed in natural ECM, heparan sulfates perform this function through 
providing binding sites for various heparin-binding growth factors. Heparin inclusion in scaffold systems improved its interaction with growth factors and bioactivity such as angiogenic performance (Rajangam et al., 2006). Functional groups from heparin improved growth factor binding capability of otherwise poorly performing material (Freeman et al., 2008). Other ECM proteins also have been proposed to have such binding sites (Martino and Hubbell, 2010). Some growth factors have domains allowing them to bind to various ECM proteins (Martino et al., 2014). Other growth factors can be engineered to carry that same domain to make them affine to ECM proteins (Martino et al., 2014).

PA nanofibers are versatile systems in terms of conjugation of binding sites for growth factors. Recently, we have designed a PA system carrying a specific arrangement of functional groups $\left(-\mathrm{COOH},-\mathrm{OH}\right.$, and $\left.-\mathrm{SO}_{3}\right)$ on nanofibers (Mammadov et al., 2011). This nanofiber system showed better growth factor binding, angiogenesis, and neurogenesis than control PA nanofibers (Mammadov et al., 2011, 2012a, 2012b). They showed significant capability to bind growth factors taking role in wound healing such as HGF, VEGF, and FGF-2 (Mammadov et al., 2012b). Hence, this nanofiber system might accelerate wound healing mechanisms such as reepithelialization and angiogenesis. Besides facilitating the performance of the exogenously delivered growth factors, these materials might also bind to endogenously secreted growth factors and minimize requirement for the growth factors in wound healing. In addition to chemical functional groups, peptide sequences could be developed for facilitating binding of other growth factors to the nanofiber system. By using phage display system, Shah et al. discovered the peptide sequence for the optimal TGF- $\beta$ binding to peptide nanofibers (Shah et al., 2010). The peptide nanofibers carrying this sequence increased performance of the TGF- $\beta$ and eventually enhanced in vivo cartilage regeneration.

Besides designing the PA to bind to growth factors, they can also be engineered to carry bioactive domains of the growth factors. Receptorbinding regions of the growth factors are generally short peptide sequences, which can be attached into the PA. In this way, the cost of using whole growth factors can be eliminated, and the unwanted effects of other parts of growth factors can be reduced. Also, aggregation of the receptor-binding domains on nanofibers might have synergistic effect on induction of signaling pathways. For example, VEGF-mimetic peptide incorporated onto PAs and presented on nanofibers induced in vivo angiogenesis stronger than same peptide delivered in soluble form (Webber et al., 2011). Receptor-binding domains of other important growth factors in wound healing can be engineered onto PA to induce various signaling pathways. 
5.3.2.2 Epitopes for Cell Adhesion One of the salient features of the wound healing mechanism is infiltration of damaged site by immense amounts of cells. The cell adhesion peptide epitopes derived from natural ECM elements (e.g., RGD or YIGSR) have been incorporated into materials in different studies to facilitate cell adhesion in damaged area. RGD incorporated into an interpenetrating network of gelatin increased cellularity and ECM organization when compared to RGD lacking material (Waldeck et al., 2007). These short peptide sequences can be easily conjugated into the PA nanofibers to facilitate cell attachment. Besides signaling purpose, this will also increase availability of the cells in material and also in damaged area, which will increase probability of their interaction with the growth factors tethered into the material. Materials with such multiple components would have synergistic effects on wound healing. Besides using peptide sequences such as RGD, which have target receptors in many cell types, specific peptide sequences can also be used to make materials bind to specific cell types (e.g., REDV sequence binds only to endothelial cells). We can change epitope density on nanofibers and obtain optimal designs for cell adhesion through dilution of adhesion epitope carrying PA molecule among control PA.

\subsubsection{Adjustment of Mechanical Properties Mechanosensation of} cells and tissues recently has gained increased interest after several studies showing the effect of substrate stiffness on cellular behavior. MSC have been shown to differentiate into neurons, muscle, and bone according to the stiffness of underlying substrate (Engler et al., 2006). The skin is also a mechanosensitive tissue (Evans et al., 2013). Keratinocytes have been shown to proliferate and migrate better on stiff substrates while differentiate better on soft substrates (Wang et al., 2012). These studies suggest that stiffness of the materials designed with purpose of wound healing will influence this very process. Better control on material stiffness will provide flexibility in the design of materials according to purpose. Various ways have been suggested to control the stiffness of PA nanofiber gels. Storage modulus increases with concentration of PA molecules (Dagdas et al., 2011). $\mathrm{Ca}^{2+}$-mediated gelation produced gels with higher stiffness values than $\mathrm{pH}$-mediated gelation, probably owing to interfiber crosslinking with $\mathrm{Ca}^{2+}$ ion (Dagdas et al., 2011). Increase in $\mathrm{Ca}^{2+}$ concentration caused increase in gel stiffness until PA neutralization has been reached (Stendahl et al., 2006). Also, PA gel moduli can be changed by type and valency of metal ions used to induce gel formation (Stendahl et al., 2006). PA molecules functionalized with 3,4-dihydroxy-L-phenylalanine (DOPA) residues showed increase in storage moduli in the order of magnitude 
when compared to ordinary PA gel (Ceylan et al., 2013). $\mathrm{Fe}^{3+}$-mediated crosslinking of DOPA molecules allowed reversible gel formation and also gained self-healing property to gels. Besides the aforementioned ways, PA molecules can also be combined with polymer molecules to give them stronger mechanical properties. To conclude, PA nanofiber materials allow control of stiffness for wound healing purposes.

\section{REFERENCES}

Balakrishnan, B., Mohanty, M., Umashankar, P.R., and Jayakrishnan, A. (2005). Evaluation of an in situ forming hydrogel wound dressing based on oxidized alginate and gelatin. Biomaterials 26, 6335-6342.

Barnea, Y., Weiss, J., and Gur, E. (2010). A review of the applications of the hydrofiber dressing with silver (Aquacel $\mathrm{Ag}$ ) in wound care. Ther Clin Risk Manag 6, 21-27.

Bayat, A., McGrouther, D.A., and Ferguson, M.W.J. (2003). Skin scarring. Br Med J 326, 88-92.

Beck, L.S., Deguzman, L., Lee, W.P., Xu, Y., McFatridge, L.A., and Amento, E.P. (1991). TGF-beta 1 accelerates wound healing: reversal of steroid-impaired healing in rats and rabbits. Growth Factors 5, 295-304.

Beer, H.D., Florence, C., Dammeier, J., McGuire, L., Werner, S., and Duan, D.R. (1997). Mouse fibroblast growth factor 10: cDNA cloning, protein characterization, and regulation of mRNA expression. Oncogene 15, 2211-2218.

Bhora, F.Y., Dunkin, B.J., Batzri, S., Aly, H.M., Bass, B.L., Sidawy, A.N., and Harmon, J.W. (1995). Effect of growth factors on cell proliferation and epithelialization in human skin. J Surg Res 59, 236-244.

Bianchi, J. (2001). Iodoflex and Iodosorb in the treatment of venous leg ulcers. Br J Nurs 10, 342-346.

Bishop, A. (2008). Role of oxygen in wound healing. J Wound Care 17, 399-402.

Bonham, P.A. (2003). Assessment and management of patients with venous, arterial, and diabetic/neuropathic lower extremity wounds. AACN Clin Issues 14, 442-456; quiz 548-450.

Bosman, F.T. and Stamenkovic, I. (2003). Functional structure and composition of the extracellular matrix. J Pathol 200, 423-428.

Boyce, S.T., Greenhalgh, D.G., Kagan, R.J., Housinger, T., Sorrell, J.M., Childress, C.P., Rieman, M., and Warden, G.D. (1993). Skin anatomy and antigen expression after burn wound closure with composite grafts of cultured skin cells and biopolymers. Plast Reconstr Surg 91, 632-641.

Brem, H. and Tomic-Canic, M. (2007). Cellular and molecular basis of wound healing in diabetes. J Clin Invest 117, 1219-1222. 
Brockes, J.P., Kumar, A., and Velloso, C.P. (2001). Regeneration as an evolutionary variable. J Anat 199, 3-11.

Carmeliet, P. (2003). Angiogenesis in health and disease. Nat Med 9, 653-660.

Centanni, J.M., Straseski, J.A., Wicks, A., Hank, J.A., Rasmussen, C.A., Lokuta, M.A., Schurr, M.J., Foster, K.N., Faucher, L.D., Caruso, D.M., et al. (2011). StrataGraft skin substitute is well-tolerated and is not acutely immunogenic in patients with traumatic wounds: results from a prospective, randomized, controlled dose escalation trial. Ann Surg 253, 672-683.

Ceylan, H., Urel, M., Erkal, T.S., Tekinay, A.B., Dana, A., and Guler, M.O. (2013). Mussel inspired dynamic cross-linking of self-healing peptide nanofiber network. Adv Funct Mater 23, 2081-2090.

Chan, R.K., Liu, P.H., Pietramaggiori, G., Ibrahim, S.I., Hechtman, H.B., and Orgill, D.P. (2006). Effect of recombinant platelet-derived growth factor (Regranex) on wound closure in genetically diabetic mice. J Burn Care Res 27, 202-205.

Choi, J.S., Leong, K.W., and Yoo, H.S. (2008). In vivo wound healing of diabetic ulcers using electrospun nanofibers immobilized with human epidermal growth factor (EGF). Biomaterials 29, 587-596.

Clark, R.A., Lanigan, J.M., DellaPelle, P., Manseau, E., Dvorak, H.F., and Colvin, R.B. (1982). Fibronectin and fibrin provide a provisional matrix for epidermal cell migration during wound reepithelization. J Invest Dermatol 79, 264-269.

Cohn, S.M., Lopez, P.P., Brown, M., Namias, N., Jackowski, J., Li, P., Mishkin, D., Lopez, J.M., and Group, U.o.M.W.S. (2004). Open surgical wounds: how does Aquacel compare with wet-to-dry gauze? J Wound Care 13, 10-12.

Dagdas, Y.S., Tombuloglu, A., Tekinay, A.B., Dana, A., and Guler, M.O. (2011). Interfiber interactions alter the stiffness of gels formed by supramolecular selfassembled nanofibers. Soft Matter 7, 3524-3532.

Dai, T., Tanaka, M., Huang, Y.Y., and Hamblin, M.R. (2011). Chitosan preparations for wounds and burns: antimicrobial and wound-healing effects. Expert Rev Anti Infect Ther 9, 857-879.

Darby, I., Skalli, O., and Gabbiani, G. (1990). Alpha-smooth muscle actin is transiently expressed by myofibroblasts during experimental wound healing. Lab Invest 63, 21-29.

Defloor, T. (1999). The risk of pressure sores: a conceptual scheme. J Clin Nurs 8 , 206-216.

Eckes, B., Nischt, R., and Krieg, T. (2010). Cell-matrix interactions in dermal repair and scarring. Fibrogenesis Tissue Repair 3, 4.

Eming, S.A., Brachvogel, B., Odorisio, T., and Koch, M. (2007a). Regulation of angiogenesis: wound healing as a model. Prog Histochem Cytochem 42, 115-170.

Eming, S.A., Krieg, T., and Davidson, J.M. (2007b). Inflammation in wound repair: molecular and cellular mechanisms. J Invest Dermatol 127, 514-525. 
Engler, A.J., Sen, S., Sweeney, H.L., and Discher, D.E. (2006). Matrix elasticity directs stem cell lineage specification. Cell 126, 677-689.

Evans, N.D., Oreffo, R.O., Healy, E., Thurner, P.J., and Man, Y.H. (2013). Epithelial mechanobiology, skin wound healing, and the stem cell niche. J Mech Behav Biomed Mater 28, 397-409.

Ferguson, M.W., Whitby, D.J., Shah, M., Armstrong, J., Siebert, J.W., and Longaker, M.T. (1996). Scar formation: the spectral nature of fetal and adult wound repair. Plast Reconstr Surg 97, 854-860.

Fitzgerald, R.H. and Steinberg, J. (2009). Collagen in wound healing: are we onto something new or just repeating the past? Foot Ankle Online J 2, 3.

Freeman, I., Kedem, A., and Cohen, S. (2008). The effect of sulfation of alginate hydrogels on the specific binding and controlled release of heparin-binding proteins. Biomaterials 29, 3260-3268.

Gabbiani, G. (2003). The myofibroblast in wound healing and fibrocontractive diseases. J Pathol 200, 500-503.

Galiano, R.D., Tepper, O.M., Pelo, C.R., Bhatt, K.A., Callaghan, M., Bastidas, N., Bunting, S., Steinmetz, H.G., and Gurtner, G.C. (2004). Topical vascular endothelial growth factor accelerates diabetic wound healing through increased angiogenesis and by mobilizing and recruiting bone marrow-derived cells. Am J Pathol 164, 1935-1947.

Garcia-Filipe, S., Barbier-Chassefiere, V., Alexakis, C., Huet, E., Ledoux, D., Kerros, M.E., Petit, E., Barritault, D., Caruelle, J.P., and Kern, P. (2007). RGTA OTR4120, a heparan sulfate mimetic, is a possible long-term active agent to heal burned skin. J Biomed Mater Res A 80, 75-84.

Gardner, S.E., Frantz, R.A., Troia, C., Eastman, S., MacDonald, M., Buresh, K., and Healy, D. (2001). A tool to assess clinical signs and symptoms of localized infection in chronic wounds: development and reliability. Ostomy Wound Manage 47, 40-47.

Gillitzer, R. and Goebeler, M. (2001). Chemokines in cutaneous wound healing. J Leukoc Biol 69, 513-521.

Goldin, A., Beckman, J.A., Schmidt, A.M., and Creager, M.A. (2006). Advanced glycation end products: sparking the development of diabetic vascular injury. Circulation 114, 597-605.

Greenhalgh, D.G. (1998). The role of apoptosis in wound healing. Int J Biochem Cell Biol 30, 1019-1030.

Gurtner, G.C., Werner, S., Barrandon, Y., and Longaker, M.T. (2008). Wound repair and regeneration. Nature 453, 314-321.

Halim, A.S., Khoo, T.L., and Mohd Yussof, S.J. (2010). Biologic and synthetic skin substitutes: an overview. Indian J Plast Surg 43, S23-S28.

Hansbrough, J.F., Achauer, B., Dawson, J., Himel, H., Luterman, A., Slater, H., Levenson, S., Salzberg, C.A., Hansbrough, W.B., and Doré, C. (1995). 
Wound healing in partial-thickness burn wounds treated with collagenase ointment versus silver sulfadiazine cream. J Burn Care Rehabil 16, 241-247.

Hashimoto, T., Suzuki, Y., Tanihara, M., Kakimaru, Y., and Suzuki, K. (2004). Development of alginate wound dressings linked with hybrid peptides derived from laminin and elastin. Biomaterials 25, 1407-1414.

Heldin, C.H. and Westermark, B. (1999). Mechanism of action and in vivo role of platelet-derived growth factor. Physiol Rev 79, 1283-1316.

Jacinto, A., Martinez-Arias, A., and Martin, P. (2001). Mechanisms of epithelial fusion and repair. Nat Cell Biol 3, E117-E123.

Jayakumar, R., Prabaharan, M., Nair, S.V., and Tamura, H. (2010). Novel chitin and chitosan nanofibers in biomedical applications. Biotechnol Adv 28, $142-150$.

Jiang, C.K., Magnaldo, T., Ohtsuki, M., Freedberg, I.M., Bernerd, F., and Blumenberg, M. (1993). Epidermal growth factor and transforming growth factor alpha specifically induce the activation- and hyperproliferation-associated keratins 6 and 16. Proc Natl Acad Sci U S A 90, 6786-6790.

Jones, I., Currie, L., and Martin, R. (2002). A guide to biological skin substitutes. Br J Plast Surg 55, 185-193.

Kim, D.H., Provenzano, P.P., Smith, C.L., and Levchenko, A. (2012). Matrix nanotopography as a regulator of cell function. J Cell Biol 197, 351-360.

Kirker, K.R., Luo, Y., Nielson, J.H., Shelby, J., and Prestwich, G.D. (2002). Glycosaminoglycan hydrogel films as bio-interactive dressings for wound healing. Biomaterials 23, 3661-3671.

Komori, M., Tomizawa, Y., Takada, K., and Ozaki, M. (2005). A single local application of recombinant human basic fibroblast growth factor accelerates initial angiogenesis during wound healing in rabbit ear chamber. Anesth Analg 100, 830-834.

Kumar, V.A., Taylor, N.L., Jalan, A.A., Hwang, L.K., Wang, B.K., and Hartgerink, J.D. (2014). A nanostructured synthetic collagen mimic for hemostasis. Biomacromolecules 15, 1484-1490.

Larjava, H., Koivisto, L., and Häkkinen, L. (2000). Keratinocyte Interactions with Fibronectin During Wound Healing In Cell Invasion, J. Heino and V.-M. Kähäri, eds. (Landes Bioscience Georgetown, TX).

Lau, K., Paus, R., Tiede, S., Day, P., and Bayat, A. (2009). Exploring the role of stem cells in cutaneous wound healing. Exp Dermatol 18, 921-933.

Levenson, S.M., Geever, E.F., Crowley, L.V., Oates, J.F., 3rd, Berard, C.W., and Rosen, H. (1965). The healing of rat skin wounds. Ann Surg 161, 293-308.

Linden, E., Cai, W., He, J.C., Xue, C., Li, Z., Winston, J., Vlassara, H., and Uribarri, J. (2008). Endothelial dysfunction in patients with chronic kidney disease results from advanced glycation end products (AGE)-mediated inhibition of endothelial 
nitric oxide synthase through RAGE activation. Clin J Am Soc Nephrol 3, 691-698.

Lipsky, B.A., Berendt, A.R., Cornia, P.B., Pile, J.C., Peters, E.J., Armstrong, D.G., Deery, H.G., Embil, J.M., Joseph, W.S., Karchmer, A.W., et al. (2012). 2012 Infectious Diseases Society of America clinical practice guideline for the diagnosis and treatment of diabetic foot infections. Clin Infect Dis 54, e132-e173.

Lobmann, R., Ambrosch, A., Schultz, G., Waldmann, K., Schiweck, S., and Lehnert, H. (2002). Expression of matrix-metalloproteinases and their inhibitors in the wounds of diabetic and non-diabetic patients. Diabetologia 45, 1011-1016.

Lodish, H., Berk, A., Zipursky, S.L., Matsudaira, P., Baltimore, D., and Darnell, J. (2000). Molecular Cell Biology, 4th edn. (W. H. Freeman New York).

Lovvorn, H.N., 3rd, Cheung, D.T., Nimni, M.E., Perelman, N., Estes, J.M., and Adzick, N.S. (1999). Relative distribution and crosslinking of collagen distinguish fetal from adult sheep wound repair. J Pediatr Surg 34, 218-223.

Lynch, S.E., Nixon, J.C., Colvin, R.B., and Antoniades, H.N. (1987). Role of platelet-derived growth factor in wound healing: synergistic effects with other growth factors. Proc Natl Acad Sci U S A 84, 7696-7700.

Malinda, K.M., Wysocki, A.B., Koblinski, J.E., Kleinman, H.K., and Ponce, M.L. (2008). Angiogenic laminin-derived peptides stimulate wound healing. Int J Biochem Cell Biol 40, 2771-2780.

Mammadov, R., Mammadov, B., Toksoz, S., Aydin, B., Yagci, R., Tekinay, A.B., and Guler, M.O. (2011). Heparin mimetic peptide nanofibers promote angiogenesis. Biomacromolecules 12, 3508-3519.

Mammadov, B., Mammadov, R., Guler, M.O., and Tekinay, A.B. (2012a). Cooperative effect of heparan sulfate and laminin mimetic peptide nanofibers on the promotion of neurite outgrowth. Acta Biomater 8, 2077-2086.

Mammadov, R., Mammadov, B., Guler, M.O., and Tekinay, A.B. (2012b). Growth factor binding on heparin mimetic peptide nanofibers. Biomacromolecules 13, 3311-3319.

Marchese, C., Chedid, M., Dirsch, O.R., Csaky, K.G., Santanelli, F., Latini, C., LaRochelle, W.J., Torrisi, M.R., and Aaronson, S.A. (1995). Modulation of keratinocyte growth factor and its receptor in reepithelializing human skin. J Exp Med 182, 1369-1376.

Markeson, D., Pleat, J.M., Sharpe, J.R., Harris, A.L., Seifalian, A.M., and Watt, S.M. (2015). Scarring, stem cells, scaffolds and skin repair. J Tissue Eng Regen Med 9, 649-668.

Martin, P. (1997). Wound healing-aiming for perfect skin regeneration. Science 276, 75-81.

Martino, M.M. and Hubbell, J.A. (2010). The 12th-14th type III repeats of fibronectin function as a highly promiscuous growth factor-binding domain. FASEB J 24, 4711-4721. 
Martino, M.M., Briquez, P.S., Guc, E., Tortelli, F., Kilarski, W.W., Metzger, S., Rice, J.J., Kuhn, G.A., Muller, R., Swartz, M.A., et al. (2014). Growth factors engineered for super-affinity to the extracellular matrix enhance tissue healing. Science 343, 885-888.

Mast, B.A. and Schultz, G.S. (1996). Interactions of cytokines, growth factors, and proteases in acute and chronic wounds. Wound Repair Regen 4, 411-420.

Menon, G.K. (2002). New insights into skin structure: scratching the surface. Adv Drug Deliv Rev 54(Suppl 1), S3-S17.

Miller, S.J., Burke, E.M., Rader, M.D., Coulombe, P.A., and Lavker, R.M. (1998). Re-epithelialization of porcine skin by the sweat apparatus. J Invest Dermatol 110, 13-19.

Min, S.K., Lee, S.C., Hong, S.D., Chung, C.P., Park, W.H., and Min, B.M. (2010). The effect of a laminin-5-derived peptide coated onto chitin microfibers on re-epithelialization in early-stage wound healing. Biomaterials 31, 4725-4730.

Mogoşanu, G.D. and Grumezescu, A.M. (2014). Natural and synthetic polymers for wounds and burns dressing. Int J Pharm 463, 127-136.

Mostow, E.N., Haraway, G.D., Dalsing, M., Hodde, J.P., King, D., and Group, O.V.U.S. (2005). Effectiveness of an extracellular matrix graft (OASIS wound matrix) in the treatment of chronic leg ulcers: a randomized clinical trial. J Vasc Surg 41, 837-843.

Moura, L.I., Dias, A.M., Carvalho, E., and de Sousa, H.C. (2013). Recent advances on the development of wound dressings for diabetic foot ulcer treatment-a review. Acta Biomater 9, 7093-7114.

O'Kane, S. and Ferguson, M.W. (1997). Transforming growth factor beta s and wound healing. Int J Biochem Cell Biol 29, 63-78.

Pardes, J.B., Takagi, H., Martin, T.A., Ochoa, M.S., and Falanga, V. (1995). Decreased levels of alpha 1(I) procollagen mRNA in dermal fibroblasts grown on fibrin gels and in response to fibrinopeptide B. J Cell Physiol 162, 9-14.

Phelps, E.A. and Garcia, A.J. (2009). Update on therapeutic vascularization strategies. Regen Med 4, 65-80.

Prompers, L., Schaper, N., Apelqvist, J., Edmonds, M., Jude, E., Mauricio, D., Uccioli, L., Urbancic, V., Bakker, K., Holstein, P., et al. (2008). Prediction of outcome in individuals with diabetic foot ulcers: focus on the differences between individuals with and without peripheral arterial disease. The EURODIALE Study. Diabetologia 51, 747-755.

Rajangam, K., Behanna, H.A., Hui, M.J., Han, X., Hulvat, J.F., Lomasney, J.W., and Stupp, S.I. (2006). Heparin binding nanostructures to promote growth of blood vessels. Nano Lett 6, 2086-2090.

Reiber, G.E., Vileikyte, L., Boyko, E.J., del Aguila, M., Smith, D.G., Lavery, L.A., and Boulton, A.J. (1999). Causal pathways for incident lower-extremity ulcers in patients with diabetes from two settings. Diabetes Care 22, 157-162. 
Rheinwald, J.G. and Green, H. (1977). Epidermal growth factor and the multiplication of cultured human epidermal keratinocytes. Nature 265, 421-424.

Roy, D.C., Wilke-Mounts, S.J., and Hocking, D.C. (2011). Chimeric fibronectin matrix mimetic as a functional growth- and migration-promoting adhesive substrate. Biomaterials 32, 2077-2087.

Rustad, K.C., Wong, V.W., Sorkin, M., Glotzbach, J.P., Major, M.R., Rajadas, J., Longaker, M.T., and Gurtner, G.C. (2012). Enhancement of mesenchymal stem cell angiogenic capacity and stemness by a biomimetic hydrogel scaffold. Biomaterials 33, 80-90.

Shah, M., Foreman, D.M., and Ferguson, M.W. (1995). Neutralisation of TGFbeta 1 and TGF-beta 2 or exogenous addition of TGF-beta 3 to cutaneous rat wounds reduces scarring. J Cell Sci 108 (Pt 3), 985-1002.

Shah, R.N., Shah, N.A., Del Rosario Lim, M.M., Hsieh, C., Nuber, G., and Stupp, S.I. (2010). Supramolecular design of self-assembling nanofibers for cartilage regeneration. Proc Natl Acad Sci U S A 107, 3293-3298.

Silva, G.A., Czeisler, C., Niece, K.L., Beniash, E., Harrington, D.A., Kessler, J.A., and Stupp, S.I. (2004). Selective differentiation of neural progenitor cells by high-epitope density nanofibers. Science 303, 1352-1355.

Simmons, T. and Linhardt, R. (2012). Application of Carbon Nanotubes to Wound Healing Biotechnology In Nanomaterials for Biomedicine, R. Nagarajan, ed. (American Chemical Society Washington, DC), pp. 156-174.

Singer, A.J. and Clark, R.A. (1999). Cutaneous wound healing. N Engl J Med 341, 738-746.

Stendahl, J.C., Rao, M.S., Guler, M.O., and Stupp, S.I. (2006). Intermolecular forces in the self-assembly of peptide amphiphile nanofibers. Adv Funct Mater 16, 499-508.

Stoscheck, C.M., Nanney, L.B., and King, L.E., Jr. (1992). Quantitative determination of EGF-R during epidermal wound healing. J Invest Dermatol 99, 645-649.

Supp, D.M. and Boyce, S.T. (2005). Engineered skin substitutes: practices and potentials. Clin Dermatol 23, 403-412.

Szabowski, A., Maas-Szabowski, N., Andrecht, S., Kolbus, A., Schorpp-Kistner, M., Fusenig, N.E., and Angel, P. (2000). c-Jun and JunB antagonistically control cytokine-regulated mesenchymal-epidermal interaction in skin. Cell 103, 745-755.

Tsang, M.W., Wong, W.K., Hung, C.S., Lai, K.M., Tang, W., Cheung, E.Y., Kam, G., Leung, L., Chan, C.W., Chu, C.M., et al. (2003). Human epidermal growth factor enhances healing of diabetic foot ulcers. Diabetes Care 26, 1856-1861.

Waldeck, H., Chung, A.S., and Kao, W.J. (2007). Interpenetrating polymer networks containing gelatin modified with PEGylated RGD and soluble KGF: 
synthesis, characterization, and application in in vivo critical dermal wound. J Biomed Mater Res A 82, 861-871.

Walker, D.J. (1999). Venous stasis wounds. Orthop Nurs 18, 65-74, 95.

Wallis, S., Lloyd, S., Wise, I., Ireland, G., Fleming, T.P., and Garrod, D. (2000). The alpha isoform of protein kinase $\mathrm{C}$ is involved in signaling the response of desmosomes to wounding in cultured epithelial cells. Mol Biol Cell 11, 1077-1092.

Wang, Y., Wang, G., Luo, X., Qiu, J., and Tang, C. (2012). Substrate stiffness regulates the proliferation, migration, and differentiation of epidermal cells. Burns $38,414-420$.

Webber, M.J., Tongers, J., Newcomb, C.J., Marquardt, K.T., Bauersachs, J., Losordo, D.W., and Stupp, S.I. (2011). Supramolecular nanostructures that mimic VEGF as a strategy for ischemic tissue repair. Proc Natl Acad Sci U S A 108, 13438-13443.

Werner, S. and Grose, R. (2003). Regulation of wound healing by growth factors and cytokines. Physiol Rev 83, 835-870.

WHO (April, 2014). Fact Sheets: Burns. Available at http://www.who.int/mediacentre/ factsheets/fs365/en/ (accessed on October 29, 2015).

Woo, K.M., Chen, V.J., and Ma, P.X. (2003). Nano-fibrous scaffolding architecture selectively enhances protein adsorption contributing to cell attachment. J Biomed Mater Res A 67, 531-537.

Yildirimer, L., Thanh, N.T., and Seifalian, A.M. (2012). Skin regeneration scaffolds: a multimodal bottom-up approach. Trends Biotechnol 30, 638-648.

Zhang, Y., Wang, B., Meng, X., Sun, G., and Gao, C. (2011). Influences of acidtreated multiwalled carbon nanotubes on fibroblasts: proliferation, adhesion, migration, and wound healing. Ann Biomed Eng 39, 414-426. 